\title{
Introduction to HICCS-52 Minitrack on Augmenting Human Intelligence: Artificially, Socially, and Ethically
}

\author{
Yibai Li \\ University of Scranton \\ yibai.li@scranton.edu
}

\author{
Xuefei (Nancy) Deng \\ California State University, \\ Dominguez Hills \\ ndeng@csudh.edu
}

\author{
Yichuan Wang \\ Newcastle University \\ yi-chuan.wang@newcastle.ac.uk
}

\section{Introduction}

Artificial Intelligence or AI is the theory and development of computer systems that can think and act humanly and rationally [1]. In recent years, more and more practitioners and researchers start to refer AI to "augmented intelligence" [2]. Augmented Intelligence is a new perspective to look at the artificial intelligence, social computing, machine learning, big data, data mining, and related areas. It has a clear emphasis that humanity, not machines, is the core of this scientific inquiry. And the ultimate goal of $\mathrm{AI}$ is to augment human, not to replace human. Augmented Intelligence describes a system consisting of both artificial intelligence agents and human agents, therefore opening up new research opportunities not only for researchers in technical areas such as artificial intelligence, machine learning, big data, and data mining, but also for researchers in behavioral science, social science, organization science, human-computer interaction and many other areas. This minitrack proposes that Augmented Intelligence can be studied from the following three perspectives.

\subsection{Augmenting Human Intelligence through Artificial Intelligence}

In the today's knowledge economy, knowledge and knowledge workers have played a pivotal role in the growth and innovation of businesses. The technological advancements in computer science and information systems have presented Artificial Intelligence (AI) to companies as a new type of knowledge aid in managing and growing their businesses. Viewed as technologies that can learn to replicate human intelligence, AI can enable companies to increase productivity and efficiency in their business operations by automating simple, repetitive tasks and streamlining workflows. For example, by applying machine learning to natural language processing (NLP), AI can help its human counterparts improve the workflows in sales and customer sales management by analyzing the tone of voice and verbiage used on a call. The International Data Corporation (IDC) forecasts that AI will unleash unprecedented levels of workplace productivity, boost global revenues by $\$ 121$ billion over the next five years and generate more jobs than it will replace.

Rather than simply viewing AI as systems with the goal of replicating and surpassing human intelligence, scientists and industry experts have started to view AI as systems to enhance and augment human capabilities. In healthcare, AI assists radiologists with image interpretation by bringing the relevant information out of the electronic medical record and presenting it to them in a meaningful way to better inform their clinical judgment [3]. AI also facilitates innovations and the creation of intellectual products. For example, IBM Watson helped musician Alex Da Kid create a popular song named "Not Easy" by uncovering and visualizing the most pervasive themes in people's deep emotions hidden in the past five years' culture data [4].

\subsection{Augmenting Human Intelligence through Social Computing}

Social computing is a novel computing paradigm [5] that can also augment human intelligence. Social computing artifacts such as social media, blog, social bookmarking, and wiki generates enormous amount of data, the "Big Data," which allows big data analytics to aid the decision-making process. Social computing artifacts such as crowdsourcing allow companies to tap into large scale, on-demand virtual workforce to perform digitized work. Social computing allows human to better identify, acquire, assimilate, generate, share, and use knowledge resources, thus augmenting human's social intelligence to process social data and afford sociality. At lower levels, social computing augments human's capabilities to acquire, assimilate, and/or share (i.e., storage/retrieval, transmission features) 
social information. At higher levels, social computing augments human intelligence to create/discover (e.g. mine) knowledge by using data generated during the conduct of social computing.

\subsection{Augmenting Human Intelligence through Value Sensitive Design}

Value sensitive design is a value-oriented design methodology commonly adopted in human-computer interaction; it seeks to under how human values (e.g., welfare, accountability, autonomy, freedom from bias) can be accounted for in the design of computer technologies [6, 7]. While Augmented Intelligence brought us the promising prospect to use the power of machine learning to uncover creative insights in fields such as science and healthcare, it's important for us to consider and incorporate human values in the design of AI systems. Value sensitive design framework has been proposed in the ethical considerations of micro-task crowdsourcing platform design [8]. Likewise, we should not underestimate the potential ethical concerns arising in the adoption and use of AI for intelligence augmentation. Researchers should continue to study how to avoid the risk of constructing machine intelligence that mirrors a narrow and privileged vision of society with its biases and stereotypes.

In addition to the three perspectives above, this minitrack welcomes any studies that investigate Augmented Intelligence from both technical perspectives and social behavioral perspectives. This minitrack welcomes papers in all formats including empirical studies, design, theory, theoretical framework, case studies, and etc. Potential topics include, but are not limited to:

- AI and social computing

- AI and knowledge creation

- AI and innovation

- AI, cultures and arts

- Business applications of AI

- AI and healthcare

- AI and decision making

- Value-Sensitive Design of AI

- The ethical issues in AI development

- The ethical issues in AI adoption and use

- The interface and interaction between artificial intelligence agents and human agents

- AI for sustainability

- AI as marketing tool

\section{Summary of Articles}

This mini-track presents 1 paper in HICCS-52. We introduce it briefly below.
$\mathrm{Ku}$, Chang, Wang, Chen, and Chen's [9] work develops a framework to integrate web crawlers, visual analytics, natural language processing, and deep learning techniques. The analytical framework is used to gain insights into interrelated relationships between online reviews and managerial responses. 4and 5-star hotels in London with 91,051 consumer reviews and 70,397 managerial responses were analyzed. The main contributions are threefold. First, hotel managers can prioritize response orders to gain insights into consumer reviews to make selfimprovement. Second, the visual analytics results enable travelers to stay at affordable hotels and enjoy 5 -star service. Finally, this study is among the first to integrate visual analytics, natural language processing, and deep learning models to analyze hotel reviews and managerial responses.

\section{Reference}

[1] Russell, S. J., Norvig, P., Canny, J. F., Malik, J. M., \& Edwards, D. D. Artificial intelligence: a modern approach, Vol. 2, No. 9. Upper Saddle River: Prentice hall, 2003.

[2] Inhi Su, Keynote speech at HICSS 51st, 2018 January

[3] AGFA, "Augmented intelligence: The next frontier in health imaging", Healthcare IT News, 2018. [Online]. Available: https://www.healthcareitnews .com/news/augmented-intelligence-next-frontierhealth-imaging. [Accessed: 10- Oct- 2018].

[4] IBM, "IBM Cognitive Music", IBM Watson x Spotify, 2018. [Online]. Available: https://www.ibm.com /watson/music/. [Accessed: 10- Oct- 2018].

[5] Y. Li and K. Joshi, "The state of social computing research: A literature review and synthesis using the latent semantic analysis approach", in AMCIS 2012 Proceedings, 2012.

[6] B. Friedman, "Value-sensitive design", interactions, vol. 3, no. 6, pp. 16-23, 1996.

[7] Friedman, B., and Kahn Jr., P. H. "Human Values, Ethics, and Design," in The Human-Computer Interaction Handbook: Fundamentals, Evolving Technologies and Emerging Applications, J. A. Jacko and A. Sears (eds.), Mahwah, NJ: Lawrence Erlbaum Associates, Inc., pp. 1177-1201. 2003.

[8] X. Deng, K. Joshi and R. Galliers, "The Duality of Empowerment and Marginalization in Microtask Crowdsourcing: Giving Voice to the Less Powerful Through Value Sensitive Design", MIS Quarterly, vol. 40, no. 2, pp. 279-302, 2016.

[9] Ku, C. H., Chang, Y. C., Wang, Y., Chen, C. H., \& Hsiao, S. H. Artificial Intelligence and Visual Analytics: A Deep-Learning Approach to Analyze Hotel Reviews \& Responses. In HICSS 2019 Proceedings, 2019. 